\title{
基于电磁场检测 PID 算法控制的智能车设计与实现
}

\section{Design and Implementation of Intelligent Vehicle Based on PID Algorithm Control for Electromagnetic Field Detection}

张秀才

Xiucai Zhang

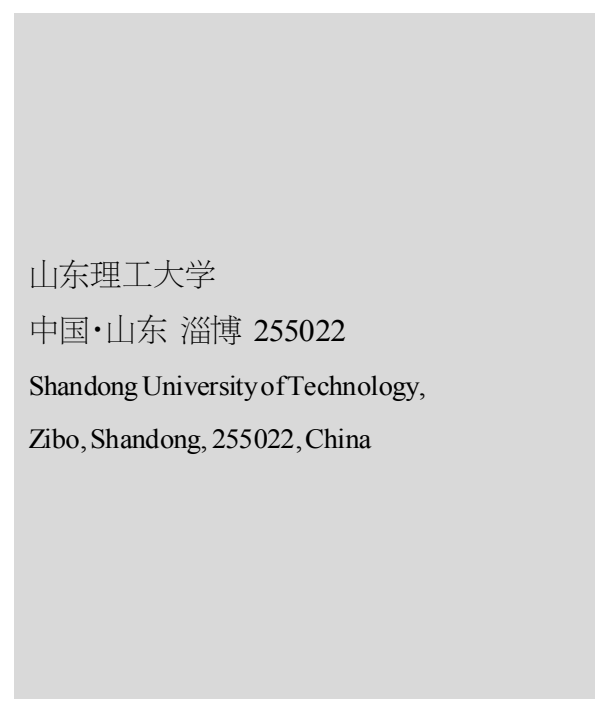

【摘要】为了能够让智能车实现更加准确的控制, 文章依据 MK60DN512ZVLQ10 微控制 系统介绍了智能车的稳压模块、运算放大器模块、 $\mathrm{H}$ 桥电路驱动模块的电路设计, 中位值 平均滤波、PD 舵机的分段控制、PID 的电机控制的算法开发流程，从而实现了智能车的信 号准确采集、舵机准确转向、电机速度的闭环控制。

【Abstract IIn order to realize more accurate control of intelligent vehicle, this paper introduces the circuit design of voltage stabilizing module, operational amplifier module and H-bridge circuit drive module of intelligent vehicle according to MK60DN512ZVLQ10 micro-control system, median value average filtering, piecewise control of PD steering gear and algorithm development flow of PID motor control. Thus, the signal acquisition of intelligent vehicle, the accurate steering of steering gear and the closed-loop control of motor speed are realized.

【关键词】电路设计; 控制算法;信号处理

【Keywords】circuit design; control algorithm; the signal processing 【DOI】10.36012/etr.v2i1.1031

\section{1 引言}

随着技术的发展, 智能出行、智能电器、智能交通[1等技术 的应用大大促进了中国制造业的飞速发展 ${ }^{[2]}$ 。如果想准确地 实现上述的工作, 可以依靠电磁寻线 PID 控制的智能车。与 其他基于黑白摄像头寻线、红外寻线的智能车相比, 电磁寻线 的智能车最大优势是受外界条件的的影响比较小。因此在传 统的循迹小车不能满足客户需求的情况下, 人们希望凭借电 磁技术的上述优势研发一种节能环保、硬件简单、数据流量且 更加稳定的循迹智能车, 最终实现自动化作业模式 ${ }^{[3]}$ 。该智能 车以 MK60DN512ZVLQ10 微控制器为核心, 硬件电路主要包 括运算放大器模块、电机驱动模块、稳压电源模块。控制小车 的算法包括运用闭环的 PID 控制算法实现对电机的控制, 利 用 PD 算法实现对舵机的转向控制、利用中位值平均滤波方 法及归一化算法对采集信号进行处理, 最终实现智能小车在 电磁线上的精确循迹。

\section{2 电路设计}

\section{1 稳定电路模块}

使用 LM2940 把电池 $7.4 \mathrm{~V}$ 的电压稳压成 $5 \mathrm{~V}$ 的电压
(见图 1), $5 \mathrm{~V}$ 电压提供给运算放大器、舵机、OLED 显示 屏、红外测距仪、编码器。然后再使用 AMS1117 芯片把 5V 的电压稳压成 3.3V (见图 2) 给 MK60DN512ZVLQ10 微控制 器供电。

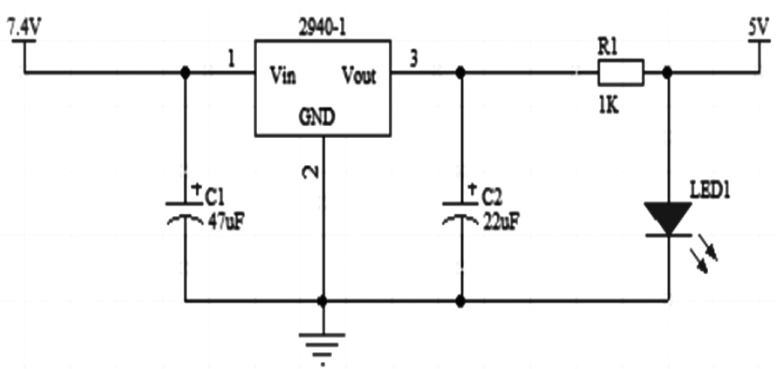

图 $17.4 \mathrm{~V}$ 稳压成 $5 \mathrm{~V}$ 电路图

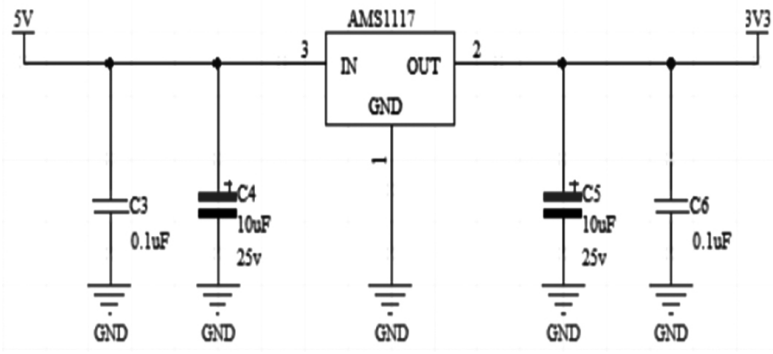

图 $25 \mathrm{~V}$ 稳压成 $3.3 \mathrm{~V}$ 电路图 
实验与研究 Experiments and Research

\section{2 电机驱动和运算放大器电路模块}

使用 OPA2350 双运算放大芯片对采集到的微弱电压进 行放大, 电路的设计如图 3 所示。其中放大的倍数 $k$ 计算公式 见式 1 。采用 $\mathrm{H}$ 桥驱动电路驱动控制直流电机, 电路设计如图 4 所示。

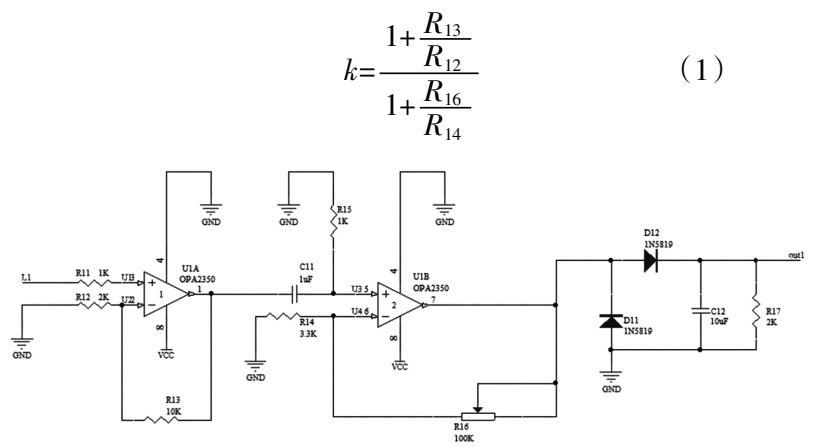

图 3 运算放大器设计电路图

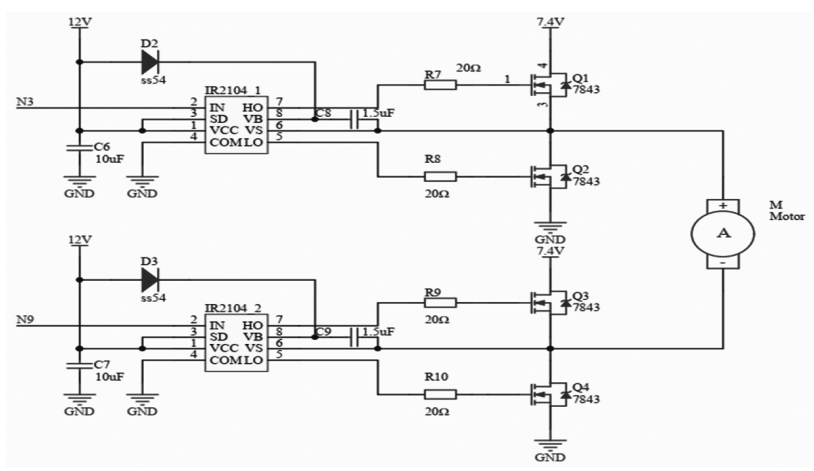

图 $4 \mathrm{H}$ 桥设计电路图

\section{3 控制算法的设计}

\section{1 中位值平均滤波算法}

通过 MK60DN512ZVLQ10 单片机每隔 3 ms 连续不断地 采集 $\mathrm{N}$ 次数据, 采用冒泡的排序方法对所采集到的数据进行 排序, 去掉一个最大值和一个最小值后, 计算 $\mathrm{N}-2$ 个数据的 算数平均数, 其中 $\mathrm{N}$ 的取值在 3 14 比较合适。此种方法能够 很好地消除因偶然出现的脉冲性干扰导致的采样值偏差, 但 是如果选取的 $N$ 太大, 则会浪费 RAM, 导致单片机的计算速 度下降。

\section{2 舵机的分段式 PD 算法}

(1)单片机首先进行读取左右两个电感采集到的电压值。 (2)计算两个电压的差值。(3)根据计算的差值 $\Delta$ 大小与所设计的 阈值 $K_{1} 、 K_{2} 、 K_{3}$ 进行比较，根据比较的值分成三个区间，不同 的区间对应着不同的 $P 、 D$ 的取值。算法流程如图 5 所示。

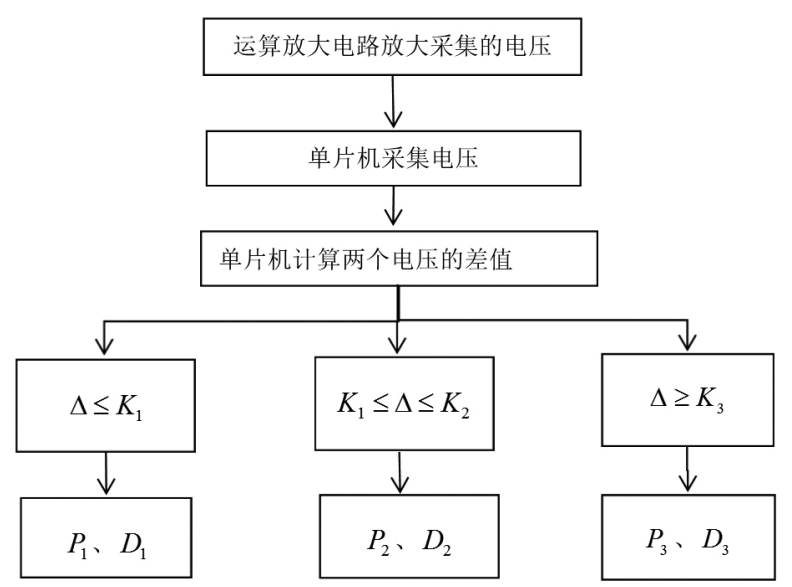

图 5 PD 算法流程图

\section{3 闭环 PID 算法对双电机进行控制}

(1) 设定一个理想的转速 $v$ 、电机最快转速 $v_{1}$ 和最低转速 的极限值 $v_{2}$ 。(2)编码器采集智能车后车轮的转速。(3)比较理想 转速与实际转速并做差值。(4)采用位置式(式 2)的 PID 算法 对下一时刻的速度计算和预测。(5)如果计算出的速度 $v \leqslant v_{1}$, 下一时刻的速度 $v=v_{1}$; 如果 $v_{1} \geqslant v_{2}$, 下一时刻的速度则为 $v_{2}$ 。(6) 通过合理的调试 PID 中的 $K p 、 K i 、 K d$ 三个参数使智能车的速 度达到反应快速、稳定、准确的目的。

$$
u(k)=K p e_{k}+K i \sum_{j=0}^{k} e_{j}+K d\left(e_{k}-e_{k-1}\right)
$$

式中, $k$ 为采样序号; $u_{k}$ 为第 $k$ 次采样时刻的单片机输出 值; $e_{k}$ 为第 $k$ 次采样时刻输入的偏差值; $e_{k-1}$ 为第 $k-1$ 次采样 时刻输入的偏差值; $K p$ 为比例系数; $K i$ 为积分系数; $K d$ 为微 分系数。

\section{4 结语}

文章从稳压电路、运算放大电路、 $\mathrm{H}$ 桥驱动电路设计、中 位值平均滤波算法、舵机的分段 PD 算法、电机的位置式 PID 算法对智能车进行了系统的设计, 根据调试的过程说明设计 的电路和所采用算法的方案是可行的。

\section{参考文献}

[1] 王孝尚.基于 STM32 的智能窗穷控制系统[J].电子世界,2017 (17): 188 .

[2] 吴爱萍,黄振兴.基于 AT89S52 的智能窗帘群控系统 [J].科技 资讯,2007(31):77-78.

[3] 赵泓扬,杨建娇. 基于 MC9S12XS128 的电磁循迹智能车控制 系统[J].常州工学院学报,2015(4):25-29. 